Bull. Korean Math. Soc. 51 (2014), No. 5, pp. 1399-1409

http://dx.doi.org/10.4134/BKMS.2014.51.5.1399

\title{
UNIFORM MODERATE DEVIATION OF SAMPLE QUANTILES AND ORDER STATISTICS
}

\author{
Shoufang Xu and Yu Miao
}

\begin{abstract}
In this article, we establish the $\mathscr{F}_{p}$-uniform moderate deviation principles of the sample quantiles and order statistics for a sequence of independent and identically distributed samples.
\end{abstract}

\section{Introduction}

First, suppose that we have an independent and identically distributed sample of size $n$ from a distribution function $F(x)$ with a continuous probability density function $f(x)$. Let $\xi_{p}$ denote the unique $p$-th quantile of $F(x)$, i.e.,

$$
\xi_{p}=\inf \{x: F(x) \geq p\}, \quad p \in(0,1) .
$$

Note that $\xi_{p}$ satisfies

$$
F\left(\xi_{p^{-}}\right) \leq p \leq F\left(\xi_{p}\right) .
$$

Let us define the sample distribution function $F_{n}(x)$ by

$$
F_{n}(x)=\frac{1}{n} \sum_{i=1}^{n} 1_{\left\{X_{i} \leq x\right\}}, \quad-\infty<x<\infty,
$$

where $1_{A}$ denotes the indicator function of the set $A$. The sample $p$-th quantile is defined as the $p$-th quantile of the sample distribution function $F_{n}(x)$ and we denote it by $\hat{\xi}_{p n}$. Thus $\hat{\xi}_{p n}$ can be represented as

$$
\hat{\xi}_{p n}=\inf \left\{x: F_{n}(x) \geq p\right\}, \quad p \in(0,1) .
$$

The quantile not only can be used for describing some properties of random variables, but also there are not the restrictions of moment conditions. As a result, it is being widely employed in diverse problems in finance, such as, quantile-hedging, optimal portfolio allocation, risk management, and so on. In practice, the large sample theory which can give the asymptotic properties of sample estimator is an important method to analyze statistical problems. There are numerous literatures to study the sample quantiles. In [6], the strong

Received March 19, 2013; Revised February 25, 2014

2010 Mathematics Subject Classification. 62G30, 60F 10.

Key words and phrases. $p$-quantile, sample $p$-quantile, order statistics, uniform moderate deviation. 
consistency of the sample quantile is given, i.e., $\hat{\xi}_{p n} \stackrel{w p 1}{\longrightarrow} \xi_{p}$. In addition, if $F(x)$ possesses a continuous density function $f(x)$ in a neighborhood of $\xi_{p}$ and $f\left(\xi_{p}\right)>0$, then

$$
\frac{n^{\frac{1}{2}} f\left(\xi_{p}\right)\left(\hat{\xi}_{p n}-\xi_{p}\right)}{[p(1-p)]^{\frac{1}{2}}} \rightarrow N(0,1) \text { as } n \rightarrow \infty,
$$

where $N(0,1)$ denotes the standard normal variable (see [6]). Suppose that $F(x)$ is twice differentiable at $\xi_{p}$, with $F^{\prime}\left(\xi_{p}\right)=f\left(\xi_{p}\right)>0$, then Bahadur [1] proved an elegant representation

$$
\hat{\xi}_{p n}=\xi_{p}+\frac{p-F_{n}\left(\xi_{p}\right)}{f\left(\xi_{p}\right)}+\widetilde{R}_{n} \text { a.e. }
$$

where $\widetilde{R}_{n}=O\left(n^{-\frac{3}{4}}(\log n)^{\frac{3}{4}}\right)$ a.e., as $n \rightarrow \infty$. Xu and Miao [7] obtained some asymptotic properties of the deviation between the sample quantiles $\hat{\xi}_{n p}$ and the quantile $\xi_{p}$ under some weak conditions. Miao et al. [5] gave the almost sure central limit theorem of the sample quantiles.

These results concentrated on the topic that the distribution function is fixed. Recently, Zielinski [8] introduced the definition of $\mathscr{F}_{p}$-uniformly strongly consistent estimator and proved that $\hat{\xi}_{p n}$ is an $\mathscr{F}_{p}$-uniformly strongly consistent estimator of $\xi_{p}$ if and only if

$$
\inf _{F \in \mathscr{F}_{p}} \min \left\{p-F\left(\xi_{p}-\epsilon\right), F\left(\xi_{p}+\epsilon\right)-p\right\}>0 \text { for every } \epsilon>0,
$$

where $\mathscr{F}_{p}$ denotes the family of all distribution functions with the unique $p$ th quantile $\xi_{p}$. Motivated by these works, we want to consider the uniform moderate deviation of the sample quantile. Under some assumptions for the function family $\mathscr{F}_{p}$, we show, in Section 2 , that the sample quantile $\hat{\xi}_{p n}$ satisfies the moderate deviation principle.

Another nature estimator of the quantile is the order statistics. Let

$$
X_{(1)} \leq X_{(2)} \leq \cdots \leq X_{(n)}
$$

denote the order statistics of the sample $\left\{X_{1}, \ldots, X_{n}\right\}$ of observations on $F(x)$. For more detail about order statistics, one can refer to David [2] and Serfling [6]. Assume that $F(x)$ is twice differentiable at $\xi_{p}$, with $F^{\prime}\left(\xi_{p}\right)=f\left(\xi_{p}\right)>0$, then as $k_{n}=n p+o\left(\sqrt{n}(\log n)^{\delta}\right)$ for some $\delta \geq \frac{1}{2}$, Bahadur [1] firstly established the following representation for order statistics

$$
X_{(k)}=\xi_{p}+\frac{k_{n} / n-F_{n}\left(\xi_{p}\right)}{f\left(\xi_{p}\right)}+R_{n} \text { a.e. }
$$

where

$$
R_{n}=O\left(n^{-3 / 4}(\log n)^{(1 / 2)(\delta+1)}\right) \text { a.e. as } n \rightarrow \infty .
$$

With respect to moderate deviation of order statistics, Miao et al. [4] obtained the asymptotic properties of the deviation between order statistics and $p$-quantile, which included large and moderate deviation and the Bahadur asymptotic efficiency. 
In general, from the Bahadur representation, some properties of sample quantile and order statistics are usually consistent. However, in Section 3, we will show that the moderate deviation principle for order statistics is different from sample quantile (there are a little different in their assumptions and representation of moderate deviation). In Section 4, we will give two examples to show that these assumptions can be satisfied.

\section{2. $\mathscr{F}_{p}$-uniformly moderate deviation for sample quantiles}

Theorem 2.1. For $p \in(0,1)$, let $\mathscr{F} p$ be the family of all distribution functions with the unique $p$-th quantile $\xi_{p}$. Let $X_{1}, \ldots, X_{n}$ be independent identically distributed random variables with distribution function $F(x) \in \mathscr{F}_{p}$. Assume that $F(x)$ is twice differentiable at $\xi_{p}$, with $F^{\prime}\left(\xi_{p}\right)=f\left(\xi_{p}\right)>0$. Let $\left\{b_{n}\right\}$ be a positive sequence satisfying

$$
b_{n} \rightarrow \infty \quad \text { and } \quad \frac{b_{n}}{\sqrt{n}} \rightarrow 0 \quad \text { as } n \rightarrow \infty .
$$

(1) If there exists a constant $\gamma>0$ such that

$$
\sup _{F \in \mathscr{F}_{p}} \sup _{x \in\left(\xi_{p}, \xi_{p}+\gamma\right)} \frac{\left|F^{\prime \prime}(x)\right|}{\left(f\left(\xi_{p}\right)\right)^{2}}<\infty,
$$

then we have

$$
\lim _{n \rightarrow \infty} \frac{1}{b_{n}^{2}} \log \sup _{F \in \mathscr{F}_{p}} P_{F}\left(\frac{\sqrt{n} f\left(\xi_{p}\right)}{b_{n}}\left(\hat{\xi}_{p n}-\xi_{p}\right) \geq r\right)=-\frac{r^{2}}{2 p(1-p)} .
$$

(2) If there exists a constant $\gamma>0$ such that

$$
\sup _{F \in \mathscr{F}_{p}} \sup _{x \in\left(\xi_{p}-\gamma, \xi_{p}\right)} \frac{\left|F^{\prime \prime}(x)\right|}{\left(f\left(\xi_{p}\right)\right)^{2}}<\infty,
$$

then we have

$$
\lim _{n \rightarrow \infty} \frac{1}{b_{n}^{2}} \log \sup _{F \in \mathscr{F}_{p}} P_{F}\left(\frac{\sqrt{n} f\left(\xi_{p}\right)}{b_{n}}\left(\hat{\xi}_{p n}-\xi_{p}\right) \leq-r\right)=-\frac{r^{2}}{2 p(1-p)} .
$$

(3) In particular, if there exists a neighborhood of $\xi_{p}$, denoted by I, such that

$$
\sup _{F \in \mathscr{F}_{p}} \sup _{x \in I} \frac{\left|F^{\prime \prime}(x)\right|}{\left(f\left(\xi_{p}\right)\right)^{2}}<\infty,
$$

then for any $r>0$, we have the following $\mathscr{F}_{p}$-uniform moderate deviation,

$$
\lim _{n \rightarrow \infty} \frac{1}{b_{n}^{2}} \log \sup _{F \in \mathscr{F}_{p}} P_{F}\left(\frac{\sqrt{n} f\left(\xi_{p}\right)}{b_{n}}\left|\hat{\xi}_{p n}-\xi_{p}\right| \geq r\right)=-\frac{r^{2}}{2 p(1-p)} .
$$

Remark 2.1. Here we need notice that the interval $\left(\xi_{p}-\gamma, \xi_{p}\right),\left(\xi_{p}, \xi_{p}+\gamma\right)$ and the neighborhood $I$ maybe dependent on the distribution function $F$.

The following lemma will be applied in our proof. 
Lemma 2.1 ([6]). Let $F$ be a distribution function. Assume that the function $F^{-1}(t), 0<t<1$, is nondecreasing and continuous, and satisfies

$$
F^{-1}(F(x)) \leq x, \quad x \in(-\infty,+\infty),
$$

and

Hence we have

$$
F\left(F^{-1}(t)\right) \geq t, \quad t \in(0,1)
$$

$$
F(x) \geq t \Leftrightarrow x \geq F^{-1}(t) .
$$

Proof of Theorem 2.1. For any $r>0$, we give the following result firstly

$$
\lim _{n \rightarrow \infty} \frac{1}{b_{n}^{2}} \log \sup _{F \in \mathscr{F}_{p}} P_{F}\left(\frac{\sqrt{n} f\left(\xi_{p}\right)}{b_{n}}\left(\hat{\xi}_{p n}-\xi_{p}\right) \geq r\right)=-\frac{r^{2}}{2 p(1-p)} .
$$

By Lemma 2.1, we have

$$
\begin{aligned}
& P_{F}\left(\frac{\sqrt{n} f\left(\xi_{p}\right)}{b_{n}}\left(\hat{\xi}_{p n}-\xi_{p}\right) \geq r\right) \\
= & \left.P_{F}\left(\frac{1}{n} \sum_{i=1}^{n} I_{\left\{X_{i} \leq \frac{b_{n} r}{\sqrt{n} f\left(\xi_{p}\right)}\right.}+\xi_{p}\right\} \leq p\right) \\
= & P_{F}\left(\sum_{i=1}^{n} I_{\left\{X_{i} \geq \frac{b_{n} r}{\sqrt{n} f\left(\xi_{p}\right)}+\xi_{p}\right\}} \geq n(1-p)\right) \\
= & P_{F}\left(\sum_{i=1}^{n} W_{n i} \geq b_{n} \sqrt{n} \delta_{n}\right),
\end{aligned}
$$

where

$$
\left.W_{n i}=I_{\left\{X_{i} \geq \frac{b_{n} r}{\sqrt{n} f\left(\xi_{p}\right)}+\xi_{p}\right\}}-E_{F} I_{\left\{X_{i} \geq \frac{b_{n} r}{\sqrt{n} f\left(\xi_{p}\right)}\right.}+\xi_{p}\right\}
$$

and

$$
\delta_{n}=\frac{n(1-p)-n E_{F} I_{\left\{X_{i} \geq \frac{b_{n} r}{\sqrt{n} f\left(\xi_{p}\right)}+\xi_{p}\right\}}}{b_{n} \sqrt{n}} .
$$

It is easy to check

$$
E_{F} I_{\left\{X_{i} \geq \frac{b_{n} r}{\sqrt{n} f\left(\xi_{p}\right)}+\xi_{p}\right\}}=1-F\left(\frac{b_{n} r}{\sqrt{n} f\left(\xi_{p}\right)}+\xi_{p}\right)
$$

and by utilizing Taylor's formula we have

(2.11) $F\left(\frac{b_{n} r}{\sqrt{n} f\left(\xi_{p}\right)}+\xi_{p}\right)=F\left(\xi_{p}\right)+F^{\prime}\left(\xi_{p}\right) \frac{b_{n} r}{\sqrt{n} f\left(\xi_{p}\right)}+\frac{1}{2} F^{\prime \prime}(\eta)\left(\frac{r b_{n}}{\sqrt{n} f\left(\xi_{p}\right)}\right)^{2}$,

where $\eta \in\left(\xi_{p}, \xi_{p}+\frac{b_{n} r}{\sqrt{n} f\left(\xi_{p}\right)}\right)$. Thus from the condition (2.1), we get

$$
F\left(\frac{b_{n} r}{\sqrt{n} f\left(\xi_{p}\right)}+\xi_{p}\right)=p+\frac{b_{n} r}{\sqrt{n}}+R_{n}
$$


where $R_{n}=o\left(\frac{b_{n}}{\sqrt{n}}\right)$ is independent of $F$. From (2.9), (2.10), (2.12), we have

$$
\delta_{n}=\frac{n(1-p)-n(1-p)+b_{n} \sqrt{n} r+o\left(b_{n} \sqrt{n}\right)}{b_{n} \sqrt{n}}=r+o(1) .
$$

Furthermore, since

$$
W_{n i}=I_{\left\{X_{i} \geq \xi_{p}+\frac{b_{n} r}{\sqrt{n} f\left(\xi_{p}\right)}\right\}}-1+F\left(\xi_{p}+\frac{b_{n} r}{\sqrt{n} f\left(\xi_{p}\right)}\right),
$$

it is easy to have

$$
E_{F}\left(W_{n i}\right)=0, \operatorname{Var}_{F}\left(W_{n i}\right)=p(1-p)+O\left(b_{n} / \sqrt{n}\right) .
$$

Through the above discussions, the equation (2.7) can be rewritten as follows

$$
P_{F}\left(\hat{\xi}_{p n} \geq \xi_{p}+\frac{b_{n} r}{\sqrt{n} f\left(\xi_{p}\right)}\right)=P_{F}\left(\frac{1}{b_{n} \sqrt{n}} \sum_{i=1}^{n} W_{n i} \geq r+o(1)\right) .
$$

Next, we prove the following Cramér functional holds: for any $\lambda \in \mathbb{R}$,

$$
\lim _{n \rightarrow \infty} \frac{1}{b_{n}^{2}} \log \sup _{F \in \mathscr{F}_{p}} E_{F} \exp \left\{\frac{\lambda b_{n}}{\sqrt{n}} \sum_{i=1}^{n} W_{n i}\right\}=\frac{\lambda^{2} p(1-p)}{2} .
$$

By Triangle inequality, we have

$$
\begin{aligned}
& \left|\frac{1}{b_{n}^{2}} \log \sup _{F \in \mathscr{F}_{p}} E_{F} \exp \left\{\frac{\lambda b_{n}}{\sqrt{n}} \sum_{i=1}^{n} W_{n i}\right\}-\frac{\lambda^{2} p(1-p)}{2}\right| \\
\leq & \frac{n}{b_{n}^{2}}\left|\log \sup _{F \in \mathscr{F}_{p}} E_{F} \exp \left\{\frac{\lambda b_{n}}{\sqrt{n}} W_{n 1}\right\}-\left(\sup _{F \in \mathscr{F}_{p}} E_{F} \exp \left\{\frac{\lambda b_{n}}{\sqrt{n}} W_{n 1}\right\}-1\right)\right| \\
& +\frac{n}{b_{n}^{2}}\left|\left(\sup _{F \in \mathscr{F}_{p}} E_{F} \exp \left\{\frac{\lambda b_{n}}{\sqrt{n}} W_{n 1}\right\}-1\right)-\frac{\lambda^{2} p(1-p) b_{n}^{2}}{2 n}\right| .
\end{aligned}
$$

By the elementary inequality:

$$
\left|e^{x}-1-x-\frac{1}{2} x^{2}\right| \leq|x|^{3} e^{|x|}, \quad \forall x \in \mathbb{R},
$$

we have

$$
\begin{aligned}
& \frac{n}{b_{n}^{2}}\left|\left(\sup _{F \in \mathscr{F}_{p}} E_{F} \exp \left\{\frac{\lambda b_{n}}{\sqrt{n}} W_{n 1}\right\}-1\right)-\frac{\lambda^{2} p(1-p) b_{n}^{2}}{2 n}\right| \\
\leq & \frac{n}{b_{n}^{2}} \sup _{F \in \mathscr{F}_{p}}\left|E_{F}\left(\exp \left\{\frac{\lambda b_{n}}{\sqrt{n}} W_{n 1}\right\}-1-\frac{1}{2}\left(\frac{\lambda b_{n}}{\sqrt{n}} W_{n 1}\right)^{2}\right)\right| \\
+ & \frac{n}{b_{n}^{2}} \sup _{F \in \mathscr{F}_{p}}\left|\frac{1}{2} E_{F}\left(\frac{\lambda b_{n}}{\sqrt{n}} W_{n 1}\right)^{2}-\frac{\lambda^{2} p(1-p) b_{n}^{2}}{2 n}\right|
\end{aligned}
$$




$$
\begin{aligned}
\leq & \frac{n}{b_{n}^{2}} \sup _{F \in \mathscr{F}_{p}} E_{F}\left(\left|\frac{\lambda b_{n}}{\sqrt{n}} W_{n 1}\right|^{3} \exp \left\{\frac{\lambda b_{n}}{\sqrt{n}}\left|W_{n 1}\right|\right\}\right) \\
& +\frac{n}{b_{n}^{2}} \sup _{F \in \mathscr{F}_{p}}\left|\frac{1}{2} E_{F}\left(\frac{\lambda b_{n}}{\sqrt{n}} W_{n 1}\right)^{2}-\frac{\lambda^{2} p(1-p) b_{n}^{2}}{2 n}\right| .
\end{aligned}
$$

Since $W_{n 1}$ is a bounded random variable, then from (2.15) and (2.18), we have (2.19) $\frac{n}{b_{n}^{2}}\left|\left(\sup _{F \in \mathscr{F}_{p}} E_{F} \exp \left\{\frac{\lambda b_{n}}{\sqrt{n}} W_{n 1}\right\}-1\right)-\frac{\lambda^{2} p(1-p) b_{n}^{2}}{2 n}\right| \rightarrow 0$ as $n \rightarrow \infty$.

Furthermore, by the fact that $W_{n 1}$ is a bounded random variable again, for enough large $n$, we have

$$
\left|\sup _{F \in \mathscr{F}_{p}} E_{F} \exp \left\{\frac{\lambda b_{n}}{\sqrt{n}} W_{n 1}\right\}-1\right| \leq \frac{1}{2} .
$$

From this estimate and the following inequalities:

$$
|\log (1+x)-x| \leq 2 x^{2} \text { for all }|x| \leq \frac{1}{2}
$$

and

$$
\left|e^{x}-1-x\right| \leq x^{2} e^{|x|} \text { for all } x \in \mathbb{R},
$$

we get

$(2.20)$

$$
\begin{aligned}
& \frac{n}{b_{n}^{2}}\left|\log \sup _{F \in \mathscr{F}_{p}} E_{F} \exp \left\{\frac{\lambda b_{n}}{\sqrt{n}} W_{n 1}\right\}-\left(\sup _{F \in \mathscr{F}_{p}} E_{F} \exp \left\{\frac{\lambda b_{n}}{\sqrt{n}} W_{n 1}\right\}-1\right)\right| \\
\leq & \frac{2 n}{b_{n}^{2}}\left(\sup _{F \in \mathscr{F}_{p}} E_{F} \exp \left\{\frac{\lambda b_{n}}{\sqrt{n}} W_{n 1}\right\}-1\right)^{2} \\
\leq & \frac{2 n}{b_{n}^{2}} \sup _{F \in \mathscr{F}_{p}}\left|E_{F} \exp \left\{\frac{\lambda b_{n}}{\sqrt{n}} W_{n 1}\right\}-1\right|^{2} \\
\leq & \frac{2 n}{b_{n}^{2}} \sup _{F \in \mathscr{F}_{p}}\left(E_{F}\left(\left(\frac{\lambda b_{n}}{\sqrt{n}} W_{n 1}\right)^{2} \exp \left\{\frac{\lambda b_{n}}{\sqrt{n}}\left|W_{n 1}\right|\right\}\right)\right)^{2} \rightarrow 0 .
\end{aligned}
$$

Hence from (2.17), (2.19) and (2.20), the limit (2.16) holds. By the GärtnerEllis theorem (see [3]), we have

$$
\lim _{n \rightarrow \infty} \frac{1}{b_{n}^{2}} \log \sup _{F \in \mathscr{F}_{p}} P_{F}\left(\frac{\sqrt{n} f\left(\xi_{p}\right)}{b_{n}}\left(\hat{\xi}_{p n}-\xi_{p}\right) \geq r\right)=-\frac{r^{2}}{2 p(1-p)} .
$$


Likewise, by Lemma 2.1, we have

$$
\begin{aligned}
& P_{F}\left(\frac{\sqrt{n} f\left(\xi_{p}\right)}{b_{n}}\left(\hat{\xi}_{p n}-\xi_{p}\right) \leq-r\right) \\
= & P_{F}\left(\frac{1}{n} \sum_{i=1}^{n} I_{\left\{X_{i} \leq \xi_{p}-\frac{b_{n} r}{\sqrt{n} f\left(\xi_{p}\right)}\right\}} \geq p\right) \\
= & P_{F}\left(\sum_{i=1}^{n} V_{n i} \geq b_{n} \sqrt{n} \delta_{n}^{\prime}\right),
\end{aligned}
$$

where

$$
V_{n i}=I_{\left\{X_{i} \leq \xi_{p}-\frac{b_{n} r}{\sqrt{n} f\left(\xi_{p}\right)}\right\}}-E_{F} I_{\left\{X_{i} \leq \xi_{p}-\frac{b_{n} r}{\sqrt{n} f\left(\xi_{p}\right)}\right\}}
$$

and

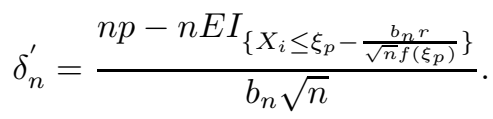

By the same proof as the term $P_{F}\left(\hat{\xi}_{p n}-\xi_{p} \geq \frac{b_{n} r}{\sqrt{n} f\left(\xi_{p}\right)}\right)$, it follows that

$$
\lim _{n \rightarrow \infty} \frac{1}{b_{n}^{2}} \log \sup _{F \in \mathscr{F}_{p}} P_{F}\left(\frac{\sqrt{n} f\left(\xi_{p}\right)}{b_{n}}\left(\hat{\xi}_{p n}-\xi_{p}\right) \leq-r\right)=-\frac{r^{2}}{2 p(1-p)} .
$$

This limit and (2.21) yield the desired results.

\section{3. $\mathscr{F}_{p}$-uniformly moderate deviation for order statistics}

In this section, we obtain the $\mathscr{F}_{p}$-uniformly moderate deviation principle of order statistics by utilizing the method to deal with the sample quantile.

Theorem 3.1. For $p \in(0,1)$, let $\mathscr{F}_{p}$ be the family of all distribution functions with the unique $p$-th quantile $\xi_{p}$. Let $X_{1}, \ldots, X_{n}$ be independent identically distributed random variables with distribution function $F(x) \in \mathscr{F}_{p}$. Assume that $F(x)$ is twice differentiable at $\xi_{p}$, with $F^{\prime}\left(\xi_{p}\right)=f\left(\xi_{p}\right)>0$. Let $\left\{b_{n}\right\}$ be a positive sequence satisfying

$$
b_{n} \rightarrow \infty \quad \text { and } \quad \frac{b_{n}}{\sqrt{n}} \rightarrow 0 \quad \text { as } n \rightarrow \infty .
$$

Then for any $r>0$, we have

(1) as $k_{n}=n(1-p)+o\left(b_{n} \sqrt{n}\right)$, if there exists a constant $\gamma>0$ such that

$$
\sup _{F \in \mathscr{F}_{p}} \sup _{x \in\left(\xi_{p}, \xi_{p}+\gamma\right)} \frac{\left|F^{\prime \prime}(x)\right|}{\left(f\left(\xi_{p}\right)\right)^{2}}<\infty,
$$

then

$$
\lim _{n \rightarrow \infty} \frac{1}{b_{n}^{2}} \log \sup _{F \in \mathscr{F}_{p}} P_{F}\left(\frac{\sqrt{n} f\left(\xi_{p}\right)}{b_{n}}\left(X_{\left(k_{n}\right)}-\xi_{p}\right) \geq r\right)=-\frac{r^{2}}{2 p(1-p)}
$$


(2) as $k_{n}=n p+o\left(b_{n} \sqrt{n}\right)$, if there exists a constant $\gamma>0$ such that

$$
\sup _{F \in \mathscr{F}_{p}} \sup _{x \in\left(\xi_{p}-\gamma, \xi_{p}\right)} \frac{\left|F^{\prime \prime}(x)\right|}{\left(f\left(\xi_{p}\right)\right)^{2}}<\infty,
$$

then

$$
\lim _{n \rightarrow \infty} \frac{1}{b_{n}^{2}} \log \sup _{F \in \mathscr{F}_{p}} P_{F}\left(\frac{\sqrt{n} f\left(\xi_{p}\right)}{b_{n}}\left(X_{\left(k_{n}\right)}-\xi_{p}\right) \leq-r\right)=-\frac{r^{2}}{2 p(1-p)} .
$$

Remark 3.1. From the proof of Theorem 3.1, we know that the two assumptions $k_{n}=n(1-p)+o\left(b_{n} \sqrt{n}\right), k_{n}=n p+o\left(b_{n} \sqrt{n}\right)$ are technical conditions in some sense, so the following standard moderate deviation can not be obtained.

$$
\lim _{n \rightarrow \infty} \frac{1}{b_{n}^{2}} \log \sup _{F \in \mathscr{F}_{p}} P_{F}\left(\frac{\sqrt{n} f\left(\xi_{p}\right)}{b_{n}}\left|X_{\left(k_{n}\right)}-\xi_{p}\right| \geq r\right)=-\frac{r^{2}}{2 p(1-p)} .
$$

In particular, if $p=\frac{1}{2}$, then (3.5) holds, i.e.,

$$
\lim _{n \rightarrow \infty} \frac{1}{b_{n}^{2}} \log \sup _{F \in \mathscr{F}_{p}} P_{F}\left(\frac{\sqrt{n} f\left(\xi_{\frac{1}{2}}\right)}{b_{n}}\left|X_{\left(k_{n}\right)}-\xi_{\frac{1}{2}}\right| \geq r\right)=-2 r^{2} .
$$

Corollary 3.1. Under the assumptions of Theorem 3.1, for $p>\frac{1}{2}$, if there exists a neighborhood of $\xi_{p}$, denoted by $I$, such that

$$
\sup _{F \in \mathscr{F}_{p}} \sup _{x \in I} \frac{\left|F^{\prime \prime}(x)\right|}{\left(f\left(\xi_{p}\right)\right)^{2}}<\infty,
$$

then for any $r>0$, we have

$$
\lim _{n \rightarrow \infty} \frac{1}{b_{n}^{2}} \log \sup _{F \in \mathscr{F}_{p}} P_{F}\left(\frac{\sqrt{n} f\left(\xi_{p}\right)}{b_{n}}\left|X_{\left(k_{n}\right)}-\xi_{p}\right| \geq r\right)=-\frac{r^{2}}{2 p(1-p)},
$$

where $k_{n}$ satisfies $k_{n}=n p+o\left(b_{n} \sqrt{n}\right)$.

Proof of Theorem 3.1. Firstly we give the proof of (3.2). It is easy to see that

$$
\begin{aligned}
& P_{F}\left(\frac{\sqrt{n} f\left(\xi_{p}\right)}{b_{n}}\left(X_{\left(k_{n}\right)}-\xi_{p}\right) \geq r\right) \\
= & P_{F}\left(X_{\left(k_{n}\right)} \geq \frac{b_{n} r}{\sqrt{n} f\left(\xi_{p}\right)}+\xi_{p}\right) \\
= & \left.P_{F}\left(\sum_{i=1}^{n} I_{\left\{X_{i} \geq \frac{b_{n} r}{\sqrt{n} f\left(\xi_{p}\right)}\right.}+\xi_{p}\right\} \geq k_{n}\right) \\
= & P_{F}\left(\sum_{i=1}^{n} W_{n i} \geq b_{n} \sqrt{n} t_{n}\right),
\end{aligned}
$$

where the sequence $\left\{W_{n i}, 1 \leq i \leq n\right\}$ is defined in (2.8) and

$$
t_{n}=\frac{k_{n}-n E_{F} I_{\left\{X_{i} \geq \frac{b_{n} r}{\sqrt{n} f\left(\xi_{p}\right)}+\xi_{p}\right\}}}{b_{n} \sqrt{n}}=r+o(1)
$$


as $k_{n}=n(1-p)+o\left(b_{n} \sqrt{n}\right)$. Hence by the same proof of Theorem 2.1, we can obtain the desired result. Next we show (3.4). Similarly, we have

$$
\begin{aligned}
& P_{F}\left(\frac{\sqrt{n} f\left(\xi_{p}\right)}{b_{n}}\left(X_{\left(k_{n}\right)}-\xi_{p}\right) \leq-r\right) \\
= & P_{F}\left(\sum_{i=1}^{n} I_{\left\{X_{i} \leq \xi_{p}-\frac{b_{n} r}{\sqrt{n} f\left(\xi_{p}\right)}\right\}} \geq k_{n}\right) \\
= & P_{F}\left(\sum_{i=1}^{n} V_{n i} \geq b_{n} \sqrt{n} t_{n}^{\prime}\right),
\end{aligned}
$$

where the sequence $\left\{V_{n i}, 1 \leq i \leq n\right\}$ is defined in (2.23) and

$$
t_{n}^{\prime}=\frac{k_{n}-n E_{F} I_{\left\{X_{i} \leq \xi_{p}-\frac{b_{n} r}{\sqrt{n} f\left(\xi_{p}\right)}\right\}}}{b_{n} \sqrt{n}}=r+o(1),
$$

as $k_{n}=n p+o\left(b_{n} \sqrt{n}\right)$. Then by the similar proof of Theorem 2.1, we can obtain the relation (3.4). So the proof of the theorem is completed.

Proof of Corollary 3.1. As the same as the proof of Theorem 3.1, it follows that if $p>\frac{1}{2}$, then $t_{n}=O\left(\sqrt{n} / b_{n}\right)$. For this case, the probability

$$
P_{F}\left(\sum_{i=1}^{n} W_{n i} \geq b_{n} \sqrt{n} t_{n}\right)
$$

could be neglected in the sense of moderate deviation. Hence, the remainder of the proof is easy.

\section{Further discussions}

In this section, we discuss the conditions (2.1), (2.3) and (2.5) by two examples.

Example 4.1. Let $X_{1}, \ldots, X_{n}$ be a sequence of independent identically distributed random variables with following distribution function

$$
F_{\lambda}(x)= \begin{cases}1-e^{-\lambda x}, & x>0, \\ 0, & x \leq 0,\end{cases}
$$

where $\lambda>0$. For $p \in(0,1)$, let $\xi_{p}$ be the $p$-th quantile of the sample distribution $F_{\lambda}(\cdot)$, then

$$
\xi_{p}=-\frac{1}{\lambda} \log (1-p) .
$$

By some simple calculating, we can see that there can exist a positive constant $\gamma$ such that

$$
\sup _{x \in\left(\xi_{p}, \xi_{p}+\gamma\right)} \frac{\left|F_{\lambda}^{\prime \prime}(x)\right|}{\left(f\left(\xi_{p}\right)\right)^{2}}=\sup _{x \in\left(\xi_{p}, \xi_{p}+\gamma\right)} \frac{e^{-\lambda x}}{(1-p)^{2}}=\frac{e^{-\lambda \xi_{p}}}{(1-p)^{2}}=\frac{1}{1-p},
$$


and

$$
\sup _{x \in\left(\xi_{p}-\gamma, \xi_{p}\right)} \frac{\left|F_{\lambda}^{\prime \prime}(x)\right|}{\left(f\left(\xi_{p}\right)\right)^{2}}=\sup _{x \in\left(\xi_{p}-\gamma, \xi_{p}\right)} \frac{e^{-\lambda x}}{(1-p)^{2}}=\frac{e^{-\lambda\left(\xi_{p}-\gamma\right)}}{(1-p)^{2}}=\frac{1}{1-p} e^{\lambda \gamma} .
$$

So if and only if $\mathscr{F}_{p}=\left\{F_{\lambda}(\cdot), \lambda \in(a, b) \subset(0, \infty)\right\}$, the conditions (2.1) and (2.3) hold. If we take $\gamma>0, \mathscr{F}_{p}=\left\{F_{\lambda}(\cdot), \lambda \in(a, b) \subset(0, \infty)\right\}$ and $I=$ $\left(\xi_{p}-\gamma, \xi_{p}+\gamma\right)$, then

$$
\sup _{F_{\lambda} \in \mathscr{F}_{p}} \sup _{x \in I} \frac{\left|F_{\lambda}^{\prime \prime}(x)\right|}{\left(f\left(\xi_{p}\right)\right)^{2}}=\sup _{\lambda \in(a, b)} \frac{1}{1-p} e^{\lambda \gamma}<\infty
$$

which implies the condition (2.5).

Example 4.2. Let $X_{1}, \ldots, X_{n}$ be a sequence of independent identically distributed normal random variables $N\left(a, \sigma^{2}\right)$, where $a \in \mathbb{R}, \sigma^{2}>0$. Let $p=\frac{1}{2}$, then $\xi_{\frac{1}{2}}=a$. Furthermore, we have $f_{a, \sigma}\left(\xi_{\frac{1}{2}}\right)=\frac{1}{\sqrt{2 \pi} \sigma}$ and

$$
\frac{\left|F_{a, \sigma}^{\prime \prime}(x)\right|}{\left(f_{a, \sigma}\left(\xi_{\frac{1}{2}}\right)\right)^{2}}=\frac{\sqrt{2 \pi}|x-a|}{\sigma} e^{-\frac{(x-a)^{2}}{2 \sigma^{2}}}
$$

where $F_{a, \sigma}(x)$ and $f_{a, \sigma}(x)$ denote the distribution function and density function of the normal random variable $N\left(a, \sigma^{2}\right)$. If we take the function set

$$
\mathscr{F}_{\frac{1}{2}}=\left\{F_{a, \sigma}(x) ; a \in \mathbb{R}, \sigma^{2}>c\right\},
$$

where $c$ is a positive constant, then it is easy to see that there exists a neighborhood $I$ (for example, $I=(a-\gamma, a+\gamma)$ for any $\gamma>0$ ) such that

$$
\sup _{F_{a, \sigma} \in \mathscr{F}_{\frac{1}{2}}} \sup _{x \in I} \frac{\left|F_{a, \sigma}^{\prime \prime}(x)\right|}{\left(f_{a, \sigma}\left(\xi_{\frac{1}{2}}\right)\right)^{2}}<\infty .
$$

Acknowledgements. The authors are very grateful to the referee for his/her valuable reports which improved the presentation of this work. This work is supported by NCET (NCET-11-0945), Plan for Scientific Innovation Talent of Henan Province (124100510014), Henan province Programs for Science and Technology Development (No. 132102310326) and Xinxiang university science and technology innovation fund (No. 12ZC07).

\section{References}

[1] R. R. Bahadur, A note on quantiles in large samples, Ann. Math. Statist. 37 (1966), no. $3,577-580$

[2] H. A. David, Order Statistics, Wiley, New York, 1981.

[3] A. Dembo and O. Zeitouni, Large Deviations Techniques and Applications, Springer, New York, 1998.

[4] Y. Miao, Y. X. Chen, and S. F. Xu, Asymptotic properties of the deviation between order statistics and p-quantile, Comm. Statist. Theory Methods. 40 (2011), no. 1, 8-14.

[5] Y. Miao, S. F. Xu, and A. Peng, Almost sure central limit theorem of sample quantiles, Adv. Decis. Sci. 2012 (2012), Article ID 671942, 7 pages. 
[6] R. J. Serfling, Approximation Theorems of Mathematical Statistics, John Wiley \& Sons, Inc., New York, 1980.

[7] S. F. Xu and Y. Miao, Limit behaviors of the deviation between the sample quantiles and the quantile, Filomat. 25 (2011), no. 2, 197-206.

[8] R. Zielinski, Uniform strong consistency of sample quantiles, Statist. Probab. Lett. 37 (1998), no. 2, 115-119.

SHOUFANG XU

Department of Mathematics and Information Science

XinXiang UNIVERSITY

Henan Province, 453000, P. R. China

E-mail address: xuxu8803@126.com

Yu MiaO

College of Mathematics and Information Science

Henan Normal University

Henan Province, 453007, P. R. China

E-mail address: yumiao728@gmail.com 